\title{
Follow up of mortality and incidence of cancer 1952-98 in men from the UK who participated in the UK's atmospheric nuclear weapon tests and experimental programmes
}

\author{
C R Muirhead, D Bingham, R G E Haylock, J A O'Hagan, A A Goodill, G L C Berridge, \\ M A English, N Hunter, G M Kendall
}

\begin{abstract}
Aims: To extend and analyse follow up of mortality and cancer incidence among men who took part in the UK's atmospheric nuclear weapon tests and experimental programmes 40-50 years ago, with particular reference to multiple myeloma and leukaemia.

Methods: A total of 21357 servicemen and male civilians from the UK who participated in the tests and a control group of 22333 male controls were followed over the period 1952-98. Analyses were conducted of mortality from various causes, and of mortality and incidence for 27 types of cancer. Results: Rates of mortality from all causes continued to be similar among test participants and controls with the longer follow up, with standardised mortality ratios (SMRs) of 89 and 88 respectively over the full follow up period. For all cancers, the corresponding SMRs were 93 for participants and 92 for controls. Mortality from multiple myeloma was consistent with national rates both for participants and controls, and the relative risk (RR) of myeloma incidence among participants relative to controls was 1.14 $190 \% \mathrm{Cl} 0.74$ to 1.74$)$ over the full follow up period and $0.79(90 \% \mathrm{Cl} 0.45$ to 1.38$)$ during the extended period of follow up (1991-98). Over the full follow up period, leukaemia mortality among participants was consistent with national rates, while rates among controls were significantly lower, and there was a suggestion of a raised risk among test participants relative to controls (RR 1.45, 90\% $\mathrm{Cl} 0.96$ to 2.17); the corresponding RR for leukaemia incidence was 1.33 (90\% Cl 0.97 to 1.84). After excluding chronic lymphatic leukaemia (CLL), which is not thought to be radiation inducible, the RR of leukaemia mortality increased to $1.83(90 \% \mathrm{Cl} 1.15$ to 2.93$)$, while that for incidence was little changed. Analysis of subgroups of participants with greater potential for exposure provided little evidence of increased risks, although the numbers of men involved were smaller and the statistical power was therefore less. Among other types of cancer, only for liver cancer incidence was there evidence of differences in rates between participants and controls in both the earlier and in the additional period of follow up. Mortality rates among test participants from causes other than cancer were generally similar to those among the controls. Conclusions: Overall levels of mortality and cancer incidence in UK nuclear weapons test participants have continued to be similar to those in a matched control group, and overall mortality has remained lower than expected from national rates. There was no evidence of an increased raised risk of multiple myeloma among test participants in recent years, and the suggestion in the first analysis of this study of a raised myeloma risk is likely to have been a chance finding. There was some evidence of a raised risk of leukaemia other than CLL among test participants relative to controls, particularly in the early years after the tests, although a small risk may have persisted more recently. This could be a chance finding, in view of low rates among the controls and the generally small radiation doses recorded for test participants. However, the possibility that test participation caused a small absolute risk of leukaemia other than CLL cannot be ruled out.
\end{abstract}

See end of article for authors' affiliations

Correspondence to Dr C R Muirhead, National Radiological Protection

Board, Chilton, Didcot Oxon OX11 ORQ, UK colin.muirhead@nrpb.org

Accepted

7 November 2002
$\mathrm{T}$ wo previous analyses examined mortality and cancer incidence among over 20000 men from the UK who took part in the UK's programmes of atmospheric nuclear weapon tests in Australia and the Pacific Ocean during the 1950s and 1960s. Both the first analysis, ${ }^{12}$ based on follow up to the end of 1983, and the second analysis, ${ }^{34}$ based on follow up to the end of 1990, suggested that test participation had no detectable effect on life expectancy or on the total risk of cancer. The first analysis found, however, an increased risk of leukaemia and multiple myeloma, both for mortality and incidence, among the test participants compared to the controls. ${ }^{12}$ It was concluded in the later, second analysis that the earlier excesses of these cancers appeared to have been chance findings, although the possibility that test participa- tion may have caused a small risk of leukaemia in the early years after the tests could not be completely ruled out. ${ }^{34}$ Studies of nuclear test veterans have also been conducted in other countries. ${ }^{56}$

During the past few years, there have been reports of raised numbers of multiple myeloma among test participants, based on records for just over 2000 British servicemen in the British Nuclear Tests Veterans Association. ${ }^{78}$ This prompted the

Abbreviations: AWE, Atomic Weapons Establishment; $\mathrm{Cl}$, confidence interval; CLL, chronic lymphatic leukaemia; ICD, International Classification of Diseases; LRF, Leukaemia Research Fund; MoD, Ministry of Defence; RR, relative risk; SMR, standardised mortality ratio 


\section{Main messages}

- Longer follow up of UK nuclear weapon test participants, up to the end of 1998 and, with roughly double the number of cancers relative to the previous analysis (up to the end of 1990), represents one of the largest studies of this kind.

- There was no evidence of an increase in overall levels of mortality and cancer incidence.

- Reports of a raised risk of multiple myeloma among test participants were not substantiated.

- There is some evidence of a raised risk of leukaemia other than CLL among test participants relative to matched controls. This could be a chance finding, but a possible effect of test participation cannot be ruled out, even in more recent years.

\section{Policy implications}

- Continued surveillance of the health of test participants may be justified, since many of these men are still alive.

present, third, analysis of the cohort of over 20000 test participants, based on follow up to the end of 1998. This paper summarises the findings; further details are given in a fuller report.' Particular attention is paid here to the patterns of rates of multiple myeloma and leukaemia, for the reasons given above. However, consideration is also given to other causes for which there were indications of differences between test participants and controls in the previous analysis, together with broad causes of death.

\section{SUBJECTS AND METHODS}

\section{Study population and radiation exposure}

The population is essentially the same as that in the second analysis. ${ }^{34}$ Contemporary records held by the Ministry of Defence (MoD) had been searched to identify test participants among servicemen and civilian employees of the Atomic Weapons Establishment (AWE) and Atomic Energy Research Establishment. These men had visited at least one of the test locations (Monte Bello Islands, Emu Field, and Maralinga Range in Australia; Malden and Christmas Islands in the Pacific Ocean) at the relevant times, or had sampled radioactive clouds. The analysis is based on 21357 test participants, of whom $29 \%$ were in the Royal Navy, $27 \%$ were in the Army, 40\% were in the Royal Air Force, and 4\% were civilians. A control group of 22333 men who did not participate in the tests was also identified from MoD archives. The controls were selected from other servicemen who served in tropical or subtropical areas and other men employed by AWE at the time of the tests. The participants and controls had very similar distributions by service and rank (see supplementary tables on the $O E M$ website), as well as by year of birth, year of enlistment or employment, and year of discharge or end of employment. ${ }^{4}$

Data from radiation dosemeters (film badges) were available for 4808 men, of whom 1716 had a recorded dose greater than zero. The mean dose from gamma radiation for men in the latter group was $9.9 \mathrm{mSv}$. In addition, the MoD indicated several groups with potential for exposure, on the basis of the duties that they performed. Further details are given in supplementary tables on the OEM website and elsewhere.

\section{Follow up}

As in the previous analyses, data on mortality and cancer incidence have been collected. Test participants and controls were followed up to 1 January 1999, using information from the NHS central registers for England, Wales, and Scotland, and the Central Services Agency in Northern Ireland, together with some other sources (see Acknowledgements). As of that time, $9 \%$ of participants and $8 \%$ of controls had emigrated ( 1882 and 1738 respectively), 23\% of both groups had died (4902 participants and 5217 controls), 68\% of participants ( 14560 ) and $69 \%$ of controls ( 15 364) were alive, and $0.1 \%$ of both groups were lost to follow up (13 and 14 respectively). Causes of death were coded according to the 9th revision of the International Classification of Diseases (ICD). ${ }^{10}$ In analysing cancer incidence, information on deaths from cancer as underlying cause was supplemented by cancer registrations and by death certificates where cancer was listed as a contributory cause. When more than one type of cancer was mentioned, leukaemia, myeloma, or lymphoma was selected in preference to other cancers in the incidence analysis. At the time that this follow up was conducted, cancer registration data were thought to be complete up to the end of 1994 . $^{11}$ Registration data up to the end of 1998 have been included in this analysis because, although these data are likely to be incomplete, cancer incidence rates in participants have been compared with rates in controls, based on the same follow up mechanism, rather than with national rates (see below).

\section{Validation}

Since it could not be assumed that all participants had been identified, information was sought previously from other sources, such as veterans' organisations. By examining the overlap between the men identified from these sources and the men identified from MoD archives, it was estimated that the main cohort of participants studied in the second analysis was $85 \%$ complete. ${ }^{4}$ Information on additional men notified by veterans' organisations subsequently has not changed this estimate. Furthermore, patterns of mortality among men notified by non-MoD sources in the period following their identification indicate that the absence of about $15 \%$ of test participants from the study cohort has not biased the findings. ${ }^{49}$

As part of the current analysis, a comparison was made of data on multiple myeloma held by the study investigators and by the University of Dundee, based on members of the British Nuclear Tests Veterans Association. ${ }^{9}$ The percentage of confirmed test participants on the Dundee list (81\%) is compatible with the value of $85 \%$ estimated previously. Furthermore, the comparison did not reveal additional death certificates or cancer registrations with multiple myeloma among test participants in the study cohort during the period for which mortality and cancer data were known to be complete.

The accuracy and completeness of haematological neoplasms collected in the study was assessed in a comparison using data from the Leukaemia Research Fund (LRF) Data Collection Study. ${ }^{12}$ The LRF maintains a registry, covering parts of England, of haematological neoplasms collected independently of data provided to the NHS central registers and which are the subject of detailed review. The comparison did not suggest any omissions of haematological neoplasms from the study, and there was good overall agreement between the LRF and study diagnoses. ${ }^{9}$

\section{Methods of analysis}

The methods of analysis were similar to those used previously. ${ }^{1-4}$ Test participants were entered into the study on the date of their first test involvement. Controls were entered on the first day such that, if they had died on that day, their death would have been included in the study. For the mortality analysis, men were regarded as being at risk until their date of death or emigration, their 85th birthday, or 1 January 1999, whichever came earliest. The same approach was used for the analysis of cancer incidence, except that men were also removed on their date of cancer registration where appropriate. 
Table 1 Observed deaths (Obs) and standardised mortality ratios (SMR) among test participants and controls and relative risks (RR) of mortality in test participants compared with controls, for broad causes of death, plus leukaemia and multiple myeloma, over the full follow up period

\begin{tabular}{|c|c|c|c|c|c|}
\hline \multirow[b]{2}{*}{ Cause of death } & \multicolumn{2}{|c|}{ Test participants } & \multicolumn{2}{|c|}{ Controls } & \multirow{2}{*}{$\begin{array}{l}\text { Mortality in test participants } \\
\text { relative to controls }\end{array}$} \\
\hline & Obs & SMR & Obs & SMR & \\
\hline All neoplasms & 1546 & $93 *$ & 1645 & $92 \dagger$ & 1.01 (0.96 to 1.08$)$ \\
\hline Leukaemia & 45 & 98 & 33 & $68 \ddagger$ & 1.45 (0.96 to 2.17$)$ \\
\hline Leukaemia excluding CLL & 40 & 106 & 23 & $58^{*}$ & 1.83 (1.15 to 2.93$)$ \\
\hline Multiple myeloma & 22 & 96 & 18 & 73 & $1.43(0.81$ to 2.54$)$ \\
\hline Other diseases & 2769 & $80 \dagger$ & 2961 & $79 \dagger$ & 1.01 (0.97 to 1.06$)$ \\
\hline Accidents and violence & 436 & $121 \dagger$ & 417 & $116^{*}$ & 1.07 (0.95 to 1.21$)$ \\
\hline Unknown & 106 & & 139 & & \\
\hline All causes & 4857 & $89 \dagger$ & 5162 & $88 \dagger$ & 1.01 (0.98 to 1.05$)$ \\
\hline
\end{tabular}

$* 0.001<p<0.01$, based on a two sided test.

$\dagger \mathrm{p}<0.001$, based on a two sided test.

$\ddagger 0.01<p<0.05$, based on a two sided test

To make comparisons with national mortality rates, expected numbers of deaths among both participants and controls were calculated by multiplying the person-years in five year age groups and for single calendar years by the corresponding mortality rates for men in England and Wales, and summing the resulting values. Standardised mortality ratios (SMRs) were calculated as the ratio of the observed to the expected number of deaths, multiplied by 100. Two sided tests for the SMRs were calculated, since both increased and decreased ratios were of interest. Tests for linear trends in SMRs by time since first test participation were based on Poisson regression. ${ }^{13}$

To compare rates among participants and controls directly, both for mortality and cancer incidence-rather than looking at ratios of SMRs - the numbers of deaths (or cancers) and person-years were stratified by age and calendar period (both in five year groups), service or employer, and by rank for those in the services or by social class for civilians. The relative risk (RR) in participants compared with controls was estimated by maximum likelihood, based-within each stratum-on a binomial distribution for the number of deaths (or cancers) among participants conditional on the total number of deaths (or cancers) among participants and controls. ${ }^{13}$ Confidence intervals (CIs) and significance levels were calculated using the associated score statistic, ${ }^{13}$ although simulations were used instead to obtain significance levels when the numbers of deaths were small. When the RR was greater than $\mathrm{l}$, a one sided significance test was performed of any increase in rates among participants compared with controls and 90\% CIs for the RRs were used, because of prior interest as to whether risks were raised among participants. When the RR was less than 1, a one sided significance test was performed of any decrease in rates among participants compared with controls. In addition, two sided tests for raised or lower RRs were performed.

Since some diseases related to multiple myeloma may not fall within the standard definition of the disease, RRs were also calculated for a wider definition of myeloma, namely ICD 9 th revision codes 203.0, 203.1, 238.6, 273.1, as derived from a review of up to date haematological information. In addition, chronic lymphatic leukaemia (CLL) is not thought to be radiation inducible, ${ }^{14}$ so this subtype was omitted from some of the leukaemia analyses. The period 2-25 years after first test participation was also studied for leukaemia, since any radiation effect may be concentrated during this time, although risks could persist subsequently. ${ }^{14}$

\section{RESULTS}

\section{Mortality by broad cause}

Table 1 shows that, over the full follow up period to the end of 1998, mortality rates in both participants and controls were lower than those in men of the same ages in England and Wales for all causes, all cancers, and all other diseases, whereas mortality rates for all accidents and violence were higher. Each of these differences was statistically significant at the 5\% level. RRs among participants compared with controls were close to $\mathrm{l}$ for each of these groupings. The RR for the incidence of all cancers was 0.99 (90\% CI 0.94 to 1.03 ), which was similar to that based on mortality data. Mortality from all causes was similar among participants and controls and was significantly lower than national rates, both up to the end of 1990 and in the following eight years, although the SMRs in both groups were higher in the later period (table 2). There were statistically significant increasing trends over time in the SMRs for all cancers and for other diseases, both for test participants and controls, due largely to low ratios within the first 10 years after entry to the study (see $O E M$ website). In contrast, the SMRs for all accidents and violence were greater than 100 among both participants and controls in the early period, but tended to decrease subsequently. Some of the following results are based on the period 10 or more years after first test participation, in order both to reduce the impact of selection into the cohort associated with the healthy worker effect, and to increase the opportunity to detect any long term effect on disease rates. Results based on the whole follow up period were generally similar. ${ }^{9}$

\section{Multiple myeloma}

Table 2 shows that, among participants relative to controls, the RR for multiple myeloma mortality based on underlying cause was 1.21 (90\% CI 0.58 to 2.53$)$ during the extended follow up period of 1991-98. Mortality for both participants and controls was consistent with national rates, during both the extended and full follow up periods (table 1). The numbers of deaths observed among participants and controls based on the wider definition of myeloma were identical to those based on the original definition. Relative to national rates, mortality among test participants was consistent with a constant level over the follow up period, although there was some variability in SMRs for specific time categories. In contrast, myeloma mortality among the controls tended to increase over time relative to national rates (see $O E M$ website).

In the analysis of myeloma incidence, there were 17 cases among participants and 10 cases among controls up to the end of 1990. These included three cases (all among participants) 
Table 2 Observed numbers of deaths and SMRs among test participants and controls, with RRs of mortality among test participants compared with controls, for selected causes of death and by calendar period

\begin{tabular}{|c|c|c|c|c|c|c|c|c|c|c|}
\hline \multirow[b]{3}{*}{ Cause of death } & \multicolumn{5}{|c|}{ Up to 31 Dec 1990} & \multicolumn{5}{|c|}{1 Jan 1991-31 Dec 1998} \\
\hline & \multicolumn{2}{|c|}{$\begin{array}{l}\text { Test } \\
\text { participants }\end{array}$} & \multicolumn{2}{|c|}{ Controls } & \multirow{2}{*}{$\begin{array}{l}\text { Mortality in test } \\
\text { participants } \\
\text { relative to controls } \\
\mathrm{RR}(90 \% \mathrm{Cl})\end{array}$} & \multicolumn{2}{|c|}{$\begin{array}{l}\text { Test } \\
\text { participants }\end{array}$} & \multicolumn{2}{|c|}{ Controls } & \multirow{2}{*}{$\begin{array}{l}\begin{array}{l}\text { Mortality in test } \\
\text { participants } \\
\text { relative to controls }\end{array} \\
\text { RR }(90 \% \mathrm{Cl})\end{array}$} \\
\hline & Obs & SMR & Obs & SMR & & Obs & SMR & Obs & SMR & \\
\hline \multicolumn{11}{|c|}{ (I) Causes with significant difference between test participants and controls in previous analysis, plus multiple myeloma } \\
\hline Leukaemia & 29 & 100 & 17 & 56 & $1.75(1.01$ to 3.06$)$ & 16 & 94 & 16 & 87 & $1.12(0.59$ to 2.13$)$ \\
\hline Leukaemia excluding CLL & 27 & 109 & 15 & 58 & 1.84 (1.02 to 3.33$)$ & 13 & 102 & 8 & 59 & $1.81(0.80$ to 4.18$)$ \\
\hline Multiple myeloma* & 9 & 77 & 6 & 48 & 1.90 (0.71 to 5.23 ) & 13 & 114 & 12 & 98 & 1.21 (0.58 to 2.53$)$ \\
\hline Cancer of mouth, tongue, pharynx & 11 & 74 & 22 & 139 & 0.56 (0.28 to 1.08$)$ & 21 & 171 & 18 & 138 & $1.26(0.71$ to 2.25$)$ \\
\hline Cancer of lung & 242 & 73 & 303 & 85 & 0.87 (0.76 to 1.01 ) & 238 & 102 & 232 & 93 & $1.10(0.94$ to 1.29$)$ \\
\hline Cancer of bladder & 29 & 106 & 11 & 37 & 2.85 (1.51 to 5.47$)$ & 23 & 85 & 23 & 79 & $1.13(0.67$ to 1.29$)$ \\
\hline Other injury and poisoning ${ }^{\dagger}$ & 132 & 130 & 107 & 104 & $1.28(1.02$ to 1.61$)$ & 34 & 131 & 35 & 127 & $1.03(0.67$ to 1.57$)$ \\
\hline \multicolumn{11}{|c|}{ (III) Other types of cancer, smoking related cancers, and broad causes of death } \\
\hline All neoplasms not in group (I) & 441 & 86 & 491 & 90 & 0.96 (0.86 to 1.07$)$ & 474 & 107 & 494 & 104 & 1.05 (0.94 to 1.17$)$ \\
\hline Neoplasms related to smoking $\ddagger$ & 389 & 82 & 462 & 90 & 0.91 (0.81 to 1.03$)$ & 384 & 104 & 395 & 100 & 1.05 (0.93 to 1.19$)$ \\
\hline All neoplasms & 761 & 82 & 850 & 86 & 0.96 (0.88 to 1.05 ) & 785 & 106 & 795 & 100 & $1.07(0.98$ to 1.17$)$ \\
\hline All diseases other than neoplasms & 1565 & 76 & 1665 & 75 & $1.02(0.96$ to 1.08$)$ & 1204 & 86 & 1296 & 86 & 1.01 (0.94 to 1.08$)$ \\
\hline Accidents and violence & 374 & 122 & 359 & 119 & 1.06 (0.94 to 1.21$)$ & 62 & 116 & 58 & 102 & $1.13(0.82$ to 1.55$)$ \\
\hline Unknown causes & 68 & & 85 & & & 38 & & 54 & & \\
\hline All causes & 2768 & 84 & 2959 & 84 & 1.00 (0.96 to 1.05 ) & 2089 & 95 & 2203 & 93 & 1.03 (0.98 to 1.08$)$ \\
\hline
\end{tabular}

*The observed numbers of deaths are unchanged for the wider definition of multiple myeloma (ICD 9th revision codes 203.0, 203.1, 238.6, and 273.1). †Other than motor vehicle traffic accidents, drowning and water transport accidents, air and space transport accidents, or suicide. $\ddagger$ Defined here as cancers of the tongue, mouth, and pharynx; oesophagus; pancreas; lung; bladder; and kidney.

notified by the NHS central registers after the previous analysis had been performed. In addition, the current analysis includes three deaths (one among participants, two among controls) with myeloma as contributory cause and another cancer as underlying cause that were classified according to the other cancer in the previous analysis. The RR among test participants relative to controls up to the end of 1990 was 2.05 ( $90 \%$ CI 0.99 to 4.30 ), which compares with 1.92 (90\% CI 0.84 to 4.50 ) as reported previously. ${ }^{4}$ During 1991-98, there were 18 cases of myeloma among participants and 25 cases among controls; the associated RR was 0.79 (90\% CI 0.45 to 1.38). As noted earlier, the ascertainment of cases after 1994 is likely to be incomplete, both for participants and controls. Over the full follow up period, myeloma incidence was similar among participants and controls (RR 1.14, 90\% CI 0.74 to 1.74 ), with 35 cases observed in both groups. These findings were little changed after adding one extra case in a participant that fell within the wider definition of myeloma, or after excluding the first 10 years after test participation.

\section{Leukaemia}

Table 2 shows that, during the extended follow up period of 1991-98, the RR based on underlying cause of death was 1.12 (90\% CI 0.59 to 2.13 ) for all leukaemias and 1.81 (90\% CI 0.80 to 4.18) after excluding CLL. Mortality among both participants and controls during the later period was consistent with national rates. Relative to national rates, leukaemia mortality among test participants was fairly constant over the follow up period (see $O E M$ website). In contrast, rates among controls increased over time, although the evidence for this trend was not as strong for leukaemia excluding CLL as for all leukaemias combined. Over the full follow up period, leukaemia mortality among test participants was consistent with national rates, whereas mortality among controls was significantly lower (table 1). Furthermore, there was stronger evidence for a raised risk among participants compared with controls when CLL was omitted (one sided $p=0.01$; two sided $\mathrm{p}=0.03$ ) than when all leukaemias were studied (one sided $p=0.07$; two sided $p=0.14$ ). There was statistically significant evidence of higher mortality rates among participants compared with controls during the period 2-25 years after first test participation, both for all leukaemias and after excluding CLL (see OEM website).

For leukaemia incidence, the number of cases up to the end of 1990 among participants was the same as reported in the previous analysis (that is, 37), ${ }^{4}$ whereas the number of cases among the control population increased from 24 to 29 , owing to the late reporting of some cases. The corresponding RR was 1.31 (90\% CI 0.84 to 2.04), which compares with 1.61 (90\% CI 1.00 to 2.57 ) as reported previously. ${ }^{4}$ During 1991-98, there were 30 leukaemia cases among participants and 24 cases among controls; the associated RR was 1.37 (90\% CI 0.86 to 2.22 ). For the incidence of leukaemia excluding CLL, the RR was 1.46 (90\% CI 0.88 to 2.45 ) up to the end of 1990 , and 1.39 (90\% CI 0.74 to 2.61 ) during the following eight years. There was some suggestion of a raised incidence over the full follow up period among participants compared with controls, both for all leukaemias (RR 1.33, 90\% CI 0.97 to 1.84 ) and after excluding CLL (RR 1.41, 90\% CI 0.96 to 2.09). Among specific leukaemia subtypes, there was a significant excess among participants relative to controls over the full follow up period for chronic myeloid leukaemia, based on 12 cases among participants and four among controls.'

\section{Other types of cancer}

Analyses of 25 other distinct types of cancer, based on the period 10 or more years after first test participation, showed that mortality among both test participants and controls was often lower than national rates, sometimes to a statistically significant extent (see OEM website). The only significant excesses relative to national rates arose for cancer of the tongue, mouth, and pharynx (among controls), malignant melanoma (among both participants and controls), and kidney cancer (among controls). There was a statistically significant difference in bladder cancer mortality between participants and controls, based on SMRs of 94 and 59 respectively. While in the previous analysis mortality rates differed significantly between test participants and controls for cancer of the tongue, mouth, and pharynx, for lung cancer, and for bladder cancer, ${ }^{3}$ during the additional follow up period mortality was similar among participants and controls for each of these causes (table 2). For the grouping of all cancers, 
Table 3 Numbers of incident cancers (I) among test participants and controls, and relative risks (RR) of incident cancer in test participants compared with controls for 27 distinct types of cancer

\begin{tabular}{|c|c|c|c|c|}
\hline \multirow[b]{2}{*}{ Type of cancer } & \multirow{2}{*}{$\begin{array}{l}\begin{array}{l}\text { Test } \\
\text { participants }\end{array} \\
\text { I }\end{array}$} & \multirow{2}{*}{$\begin{array}{l}\text { Controls } \\
\mathrm{I}\end{array}$} & \multicolumn{2}{|c|}{$\begin{array}{l}\text { Incidence rate in test } \\
\text { participants relative to } \\
\text { controls }\end{array}$} \\
\hline & & & RR & $90 \% \mathrm{Cl}^{*}$ \\
\hline Tongue, mouth, pharynx & 59 & 76 & 0.83 & 0.61 to 1.12 \\
\hline Oesophagus & 73 & 82 & 0.94 & 0.71 to 1.25 \\
\hline Stomach & 117 & 122 & 1.02 & 0.82 to 1.28 \\
\hline Large intestine and rectum & 316 & 326 & 1.02 & 0.90 to 1.17 \\
\hline Liver & 33 & 18 & 1.99 & 1.19 to 3.38 \\
\hline Primary liver cancer & 22 & 13 & 1.83 & 0.98 to 3.46 \\
\hline Gallbladder & 3 & 7 & 0.45 & 0.11 to 1.60 \\
\hline Pancreas & 77 & 75 & 1.08 & 0.81 to 1.42 \\
\hline Larynx & 49 & 57 & 0.93 & 0.66 to 1.30 \\
\hline Lung & 528 & 598 & 0.94 & 0.85 to 1.04 \\
\hline Bone & 5 & 5 & 1.16 & 0.35 to 3.86 \\
\hline Connective and soft tissue & 9 & 12 & 0.74 & 0.32 to 1.65 \\
\hline Malignant melanoma & 55 & 54 & 1.09 & 0.78 to 1.53 \\
\hline Other skin cancer & 332 & 395 & 0.89 & 0.78 to 1.01 \\
\hline Prostate & 244 & 216 & 1.22 & 1.04 to 1.43 \\
\hline Testis & 19 & 21 & 0.84 & 0.47 to 1.49 \\
\hline Bladder & 156 & 152 & 1.09 & 0.89 to 1.32 \\
\hline Kidney & 71 & 102 & 0.74 & 0.57 to 0.96 \\
\hline Tumours of central nervous system & 83 & 77 & 1.13 & 0.86 to 1.50 \\
\hline Thyroid & 6 & 3 & 1.92 & 0.51 to 7.97 \\
\hline Adrenals & 3 & 2 & 1.64 & 0.28 to 11.13 \\
\hline Hodgkin's disease & 15 & 18 & 0.81 & 0.43 to 1.54 \\
\hline Non-Hodgkin's lymphoma & 81 & 80 & 1.04 & 0.79 to 1.36 \\
\hline Multiple myeloma & 34 & 35 & 1.07 & 0.70 to 1.64 \\
\hline Multiple myeloma (wider definition) $\dagger$ & 35 & 35 & 1.11 & 0.72 to 1.69 \\
\hline Leukaemia: whole follow up period & 67 & 53 & 1.33 & 0.97 to 1.84 \\
\hline Leukaemia excluding CLL: whole follow up period & 49 & 36 & 1.41 & 0.96 to 2.09 \\
\hline Leukaemia: $2-25$ years & 29 & 10 & 3.17 & 1.63 to 6.31 \\
\hline Leukaemia excluding CLL: $2-25$ years & 23 & 6 & 3.97 & 1.73 to 9.61 \\
\hline Polycythaemia vera & 12 & 13 & 0.92 & 0.44 to 1.94 \\
\hline Other specified neoplasms & 123 & 142 & 0.92 & 0.75 to 1.14 \\
\hline Unspecified neoplasms & 76 & 103 & 0.81 & 0.63 to 1.06 \\
\hline All neoplasms excluding non-melanoma skin cancer & 2309 & 2447 & 1.00 & 0.95 to 1.05 \\
\hline All neoplasms & 2641 & 2842 & 0.99 & 0.94 to 1.03 \\
\hline
\end{tabular}

For leukaemia the whole follow up period, and the period 2-25 years after start of first test participation, are considered. For all other specific cancers the period more than 10 years after start of first test participation is considered.

${ }^{*}$ Confidence interval

†ICD 9th revision codes 203.0, 203.1, 238.6, and 273.1.

mortality among both participants and controls was significantly lower than national rates up to 1990, but was similar to national rates in the following eight years.

Table 3 shows that for most individual types of cancer, there was little evidence of differences in incidence rates between test participants and controls. However, there was a significantly raised incidence of liver cancer (one sided $p=0.012$; two sided $\mathrm{p}=0.016$ ) and prostate cancer (one sided $p=0.018$; two sided $p=0.036)$, and a significantly lower incidence of kidney cancer (one sided $\mathrm{p}=0.030$; two sided $\mathrm{p}=0.060)$ among participants compared with controls. The RR for liver cancer was similar in the previous and the additional follow up periods, whereas the evidence for a raised risk of prostate cancer incidence among participants compared with controls was stronger during 1991-98 than earlier. While in the previous analysis the incidence of bladder cancer was significantly higher among participants than controls and the incidence of non-melanoma skin cancer was significantly lower, the incidence of both was similar in the two groups over 1991-98. ${ }^{9}$ During this latter period, the RR for the incidence of all cancers combined was 1.01 (90\% CI 0.95 to 1.07 ).

\section{Mortality from causes other than cancer}

For the grouping of all diseases other than neoplasms, table 2 shows that mortality rates were similar among participants and controls and were significantly lower than national rates, both in the previous analysis and in the additional follow up period. For the grouping of all accidents and violence, mortality among participants and controls was comparable in both periods, and was consistent with national rates during 1991-98. For many specific causes other than neoplasms, including diseases related to smoking, mortality among both test participants and controls was significantly lower than or consistent with national rates, and rates were generally similar among participants and controls. ${ }^{9}$ However, SMRs were raised significantly to a similar extent among both participants and controls for diseases related to alcohol and for air accidents. Also, there was some evidence of raised mortality for a category of "other injury and poisoning" among participants relative to controls (RR $1.24,90 \%$ CI 0.99 to 1.56 ) and to national rates (SMR 126). Further examination showed that this finding could not be explained solely on the basis of one or two specific causes of death, and there was no indication of a raised risk during the additional period of follow up (table 2).

\section{Mortality and cancer incidence in test participants by type and degree of exposure}

(Note: Supplementary material relevant to this section is available on the $O E M$ website.)

Among test participants known to have been monitored for exposure to radiation, leukaemia mortality was similar to 
national rates, irrespective of whether or not a non-zero gamma dose had been recorded. There was also no indication of an increasing trend in leukaemia risk with increasing dose, either for mortality or incidence, although the numbers of cases were small. Multiple myeloma mortality was, if anything, raised relative to national rates among participants with a recorded gamma dose, and decreased among monitored participants with no recorded dose ( SMRs of 210 and 24 respectively, based on a 10 year lag); the associated RR was 16 (90\% CI 1.74 to 314 ), one sided $p=0.009$, two sided $p=0.01$. In the corresponding incidence data, the evidence for a raised myeloma risk among those with a recorded dose compared to monitored participants with no recorded dose was weaker (RR 4.91 ( $90 \%$ CI 0.94 to 26.8 ), one sided $p=0.057$, two sided $\mathrm{p}=0.08)$. Results based on the wider definition of multiple myeloma were identical. There was weak evidence of an increasing trend in multiple myeloma mortality with increasing gamma dose $(\mathrm{p}=0.094$, based on a one sided test), with slightly weaker evidence of an increasing trend for myeloma incidence $(p=0.12)$. For the grouping of all cancers other than myeloma and leukaemia, analyses of mortality and incidence by the level of recorded gamma dose showed no significant trends in the risk, although the statistical precision was low.

Among participants who were present at a major operation (as opposed to those who visited the test locations subsequently), mortality for leukaemia and multiple myeloma was consistent with national rates, and mortality from all solid cancers combined was significantly lower than national rates. RRs among participants relative to controls were generally similar in incidence and mortality analyses, and were consistent with a value of one for myeloma and leukaemia. Generally similar findings arose both for a group of 759 men identified by MoD as being liable to exposure to radiation, and for 1041 participants among whom MoD had advised that any undocumented intakes of radionuclides was most likely to have occurred. However, the numbers of myelomas and leukaemias in these two groups were small. The findings were similar when men who were in either of the above two groups or who were recorded as having a radiation dose were studied together. However, as in some of the other analyses, there was an indication of a raised leukaemia incidence relative to controls over the period 2-25 years after first test involvement. For participants not in any of the preceding categories, rates of myeloma and of all cancers other than leukaemia and myeloma were consistent with national mortality rates and with mortality and incidence rates among controls, whereas leukaemia rates tended to be raised, particularly during the period 2-25 years after first test participation. In the above analyses, RRs for multiple myeloma based on the wider definition of the disease were identical to those for the standard definition in the case of mortality and were similar in the case of incidence.

\section{DISCUSSION}

\section{General considerations}

This study design aimed to detect effects of test participation on mortality and cancer, whatever the cause. If the radiation doses recorded for participants are a fair reflection of the broad levels of exposure, it would not be expected that effects associated with such small doses could be observed. The investigators have encountered no evidence that the recorded doses are substantial underestimates. Nevertheless, they have conducted analyses that should have been able to detect raised risks if, in fact, doses to the groups most likely to have been exposed had been much larger than recorded. While it is known that radiation can induce many types of cancer, ${ }^{14}$ the identity of any other hazardous factor would depend on the disease in question. For multiple myeloma and leukaemia, there is limited information on other risk factors. ${ }^{15}$

\section{Multiple myeloma}

The current analysis was initiated because of concerns raised about a possible increased level of multiple myeloma among test participants, based on records for just over 2000 British servicemen in the British Nuclear Tests Veterans Association. $^{78}$ An intercomparison with the University of Dundee did not identify any extra myeloma registrations up to the end of 1994, nor extra deaths up to the end of 1998 with myeloma listed on the death certificate, among confirmed test participants in the study cohort. Furthermore, the coverage of participants in the cohort was similar to that estimated previously (that is, $85 \%$ ). A separate comparison with data from an independent registry of haematological diseases did not identify extra cases of myeloma among participants or controls. Thus, this analysis should provide valid comparisons of rates in participants with rates in controls and with national mortality rates.

Over the full period of follow up, there was little evidence of a raised risk of multiple myeloma among participants, relative either to national mortality rates, or to rates of myeloma mortality or incidence among controls. Comparing participants with controls, the precision of the RR was somewhat limited for mortality (1.32, 90\% CI 0.74 to 2.37 ), but the confidence interval was tighter in the corresponding incidence analysis (1.14, 90\% CI 0.74 to 1.74$)$. While the first analysis showed a significantly raised risk of myeloma in participants relative to controls, based on follow up to the end of $1983,{ }^{12}$ this finding was not repeated in the following seven year follow up, ${ }^{3}$ nor in the extended follow up presented here. Furthermore, myeloma mortality among participants during 1991-98 was similar to that expected from national rates. Results based on a wider definition of multiple myeloma were similar or identical to those based on the standard definition of the disease.

Myeloma rates were also studied in subgroups of test participants, such as those identified as being liable to exposure to radiation or in whom undocumented inhalation or ingestion of radionuclides, if any, is most likely to have occurred. Generally these analyses did not indicate raised myeloma rates, although the numbers of cases were small. Mortality among men with a recorded dose was significantly greater than that among other participants monitored for radiation exposure, although the evidence for a difference between these two groups was weaker in the corresponding incidence data. Further analysis of monitored participants showed weak evidence of an increasing trend in myeloma mortality with gamma dose, which was weaker still in the corresponding incidence data. The interpretation of these results is complicated by the small numbers of myelomas and by indications that mortality among monitored participants with no recorded gamma dose might be substantially lower than national rates.

Results from other studies are relevant in interpreting these findings. While early analyses of the Japanese atomic bomb survivors showed an association between radiation and multiple myeloma, the trend in myeloma incidence with dose was not statistically significant after a review of diagnoses. ${ }^{16}$ Results for groups with medical or occupational radiation exposures have been inconsistent, and overall, the present evidence for an association between radiation and myeloma is weak. ${ }^{14}$ Given that the recorded gamma doses for participants in the current study are generally much lower than those in the studies cited above, that the numbers of myelomas in the dose trend analysis are small, and that various subgroups have been considered, it seems likely that the results for participants with a positive gamma dose are due to chance.

Multiple myeloma rates have also been studied in other groups of nuclear weapons test participants. In the "Five Series Study" of approximately 70000 US military personnel who took part in at least one of five selected US nuclear weapons test series in Nevada or the Pacific in the 1950s, ${ }^{5}$ myeloma mortality was slightly less than national rates (based on 82 
deaths) and was compatible with that in a matched control group (RR 1.10, 95\% CI 0.79 to 1.52 ). Other studies, although smaller, also do not provide strong evidence of a raised risk of myeloma. For example, among 528 men from New Zealand who participated in UK atmospheric nuclear weapons tests in the Pacific, ${ }^{6}$ one myeloma death was observed compared with 0.3 expected from national rates.

Taken overall, this analysis does not indicate an association between participation in the UK nuclear weapons test programmes and the risk of multiple myeloma. In particular, there is no indication of an increase in myeloma rates among participants in recent years, other than would be expected as men born at the same time reach older ages. Although previous analyses of this cohort reported a raised risk of myeloma in the early years following test participation, compared to rates in the control group, it seems likely-as suggested previously ${ }^{3}$ - that this was a chance finding. Analyses of subgroups with greater potential for exposure provided little evidence of increased risks, although the numbers of men involved were smaller and the statistical power was therefore less.

\section{Leukaemia}

The first analysis of UK test participants identified a raised risk of leukaemia relative to controls, ${ }^{12}$ which is reflected here in the results for the period 2-25 years after first test participation. This finding was difficult to interpret, given that mortality in participants was slightly above national rates, whereas control rates were substantially lower. Furthermore, it is unclear which factors might give rise to such low leukaemia rates in the controls, other than perhaps chance. In the second analysis, the RR for leukaemia decreased as the SMR for the controls increased, with little indication of a raised risk during the extra follow up period of 1984-90. ${ }^{34}$

Over the additional eight years of follow up for the current analysis, mortality from leukaemia of all types among participants was similar to that expected from national rates and to that among the controls. However, during this period, there was a greater suggestion of a raised rate among test participants compared with controls when CLL was excluded from the mortality analysis or when incidence data were studied. The difference between the SMRs for all leukaemias over the full follow up period was smaller than that in the periods covered by the previous analyses (values of 98 in participants and 68 in controls), although there was still some evidence of a raised rate in participants compared with controls (RR 1.45, 90\% CI 0.96 to 2.17). The evidence for a difference in mortality was stronger after excluding CLL, although this reflected in part a low SMR for controls (that is, 58, compared with 106 for participants). The RR for leukaemia incidence was similar both to that for leukaemia mortality and for the incidence of leukaemia excluding CLL, with values of about 1.3-1.4 that fell just short of statistical significance.

Leukaemia was examined in subgroups of participants. There was no evidence of raised risks among men with a recorded dose, or of an increasing trend in risk with increasing gamma dose among monitored test participants, although the numbers of cases in these analyses were limited. Among men identified as having potential for radiation exposure, leukaemia rates were not noticeably raised relative to national rates, nor was there clear evidence that risks were higher than in test participants overall, although the data were sparse. However, particularly among test participants who were not present at a major operation, there was evidence of raised risks compared with controls, which was stronger over the period 2-25 years following test participation than over whole follow up period. This latter finding is similar to that previously reported by Darby and colleagues, ${ }^{12}$ who were unable to highlight any characteristics that distinguished these leukaemia cases from other test participants. There was no single test operation at which leukaemia risks were particularly raised, although again the small numbers limited inferences.

Most of the information linking radiation exposure of adults and leukaemia relates to relatively high doses, received either acutely or in fractions. While direct confirmation of raised leukaemia risks from low dose exposures is difficult, studies of radiation workers have indicated an association between occupational radiation exposure and the risk of leukaemia, of a magnitude consistent with that predicted from the Japanese atomic bomb data. ${ }^{17}{ }^{18}$ The doses received by participants in nuclear weapons tests are likely, in the main, to be lower than those received by radiation workers employed for many years in the nuclear industry. Furthermore, in contrast to radiation workers, only a minority of weapons test participants have recorded radiation doses, making it harder still to detect raised leukaemia risks related to radiation among these men. However, since any effect of test participation might be caused by non-radiation factors, it is important to consider results from other studies of nuclear weapon test participants. For example, in the Five Series Study of US military personnel involved in US tests in Nevada or the Pacific, ${ }^{5}$ leukaemia mortality was less than national rates, with some very weak evidence of a raised risk relative to a matched control group (RR 1.15, 95\% CI 0.93 to 1.43 ). Among men from New Zealand who participated in UK nuclear weapons tests in the Pacific, ${ }^{6}$ there were four leukaemia deaths observed compared with 0.8 expected from national rates, and the RR compared to a matched control group was 5.6 (90\% CI 1.0 to 41.7$)$.

Even though the relative difference between leukaemia rates in UK test participants and controls has narrowed with increasing follow up, there is still some evidence of a raised risk among participants relative to controls. Since mortality in controls is still low relative to national rates, the possibility of a chance finding cannot be ruled out. Nevertheless, the evidence for a raised risk appeared to be stronger when CLLwhich does not appear to be radiation inducible-was excluded from the analyses. Taken overall, the current analysis indicates that the possibility that test participation has caused a small absolute risk of leukaemia other than CLL cannot be ruled out and that, while the evidence for any risk appears to have been greatest in the early years after the tests, a small risk might have persisted in more recent years.

\section{Other cancers and non-cancer deaths}

While it is known that radiation can induce a wide range of different cancers, ${ }^{14}$ the interpretation of results for more than 20 specific cancer types requires care owing to the possibility of chance findings. There were significant differences between the participants and controls for bladder cancer (increased among participants) based on mortality data, and for liver and prostate cancer (both increased among participants) plus kidney cancer (increased among controls) based on incidence data. Only for liver cancer incidence was there evidence of differences in rates between participants and controls in both the earlier and the additional follow up periods, although the statistical significance of these results was slightly weaker when primary liver cancer was analysed. As background, studies of the Japanese atomic bomb survivors and of groups who received high radiation doses for medical reasons have shown raised risks of bladder and liver cancer, whereas the evidence for a link between radiation and prostate cancer is weaker. ${ }^{14}$ While alcohol is a risk factor for liver cancer, mortality from alcohol related diseases other than cancer was similar among test participants and controls.

Mortality for specific cancer types among both participants and controls was usually less than expected from national rates. However, for all cancers combined, mortality among both participants and controls was closer to national rates during the most recent eight years of follow up than in the period of the previous analysis, which may reflect a "wearing 
off" of the healthy worker effect at long periods after the start of employment. There was a slight suggestion that mortality from all cancers combined might be higher among participants than controls in the most recent period, but the corresponding incidence data showed similar rates in the two groups. Results for a grouping of cancers related to smoking were fairly similar to those for all cancers combined, suggesting that smoking habits have not biased the comparisons of participants and controls. Among men monitored for radiation exposure or who had potential for exposure, risks of cancers other than leukaemia and multiple myeloma were generally not raised. Thus, in line with studies of US and New Zealand test participants, ${ }^{56}$ there is very little evidence from the current analysis to indicate that test participation has influenced the induction of cancer generally.

Most of the evidence relating radiation to late health effects concerns cancer. However, follow up of the Japanese atomic bomb survivors has indicated raised mortality from noncancer diseases in recent years, ${ }^{19}$ although it is unclear whether these increases persist down to low radiation doses. In the current study, mortality was similar among participants and controls, both for non-cancer diseases in total and for specific disease groupings. This also held for diseases related to smoking, again indicating that smoking habits have not affected comparisons of the two groups. As with cancer overall, SMRs for non-cancer diseases appeared to be higher in the most recent period of follow up than in the earlier period, again pointing to a wearing off of the healthy worker effect. However, even during this recent period, mortality from all non-cancer diseases combined was still significantly less than national rates, among both participants and controls. Mortality from all accidents and violence was also similar among participants and controls, although the rates were raised relative to national rates. In common with other studies of nuclear test participants, ${ }^{56}$ there is little or no evidence from the current analysis to indicate that test participation has influenced non-cancer mortality.

\section{ACKNOWLEDGEMENTS}

The authors gratefully acknowledge the contributions made by the following organisations and individuals: Professor Nicholas Wald and other members of the Advisory Group set up to oversee this analysis (a list of members is given elsewhere ${ }^{9}$ ); the staff of MoD and AWE who assisted in the collection of data; the organisations that provided follow up information, including the Office for National Statistics, the General Register Offices for Scotland and Northern Ireland, the General Register Office of Ireland, the Benefits Agency of the Department of Social Security, the Central Services Agency of the Northern Ireland Department of Health and Social Services, the Northern Ireland Cancer Registry, the Health Departments in Dublin, Guernsey, Jersey, and the Isle of Man, MoD Medical Statistics, and AEA Technology; Sue Rabbitt Roff (University of Dundee) for taking part and sharing her data in the multiple myeloma intercomparison; Mrs Sheila Gray for representing the British Nuclear Tests Veterans Association on the Advisory Group; the Leukaemia Research Fund Centre for Clinical Epidemiology for their participation in the comparison of haematological diagnoses; and Dr Andrew Wotherspoon (Royal Marsden), for providing advice on diseases related to multiple myeloma. The substantial contributions made by Professors Sarah Darby and Sir Richard Doll (Cancer Research UK) to the setting up of this study and to conducting the first two analyses are also gratefully acknowledged.

\section{Authors' affiliations}

C R Muirhead, D Bingham, R G E Haylock, J A O'Hagan,

A A Goodill, G L C Berridge, M A English, N Hunter, G M Kendall,

National Radiological Protection Board, Chilton, Didcot, Oxon

OX11 ORQ, UK

Funding: Ministry of Defence

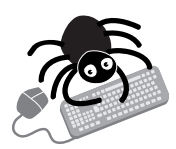

Supplementary tables can be viewed on the OEM website (www.occenvmed.com)

\section{REFERENCES}

1 Darby SC, Kendall GM, Fell TP, et al. A summary of mortality and incidence of cancer in men from the United Kingdom who participated in the United Kingdom's atmospheric nuclear weapon tests and experimental programmes. BMV 1988:296:332-8.

2 Darby SC, Kendall GM, Fell TP, et al. Mortality and cancer incidence in UK participants in UK atmospheric nuclear weapon tests and experimental programmes. Chilton, NRPB-R214. London: HMSO, 1988

3 Darby SC, Kendall GM, Fell TP, et al. Further follow-up of mortality and incidence of cancer in men from the United Kingdom who participated in the United Kingdom's atmospheric nuclear weapon tests and experimental programmes. BM 1993;307:1530-5.

4 Darby SC, Kendall GM, Fell TP, et al. Mortality and cancer incidence 1952-1990 in UK participants in the UK atmospheric nuclear weapon tests and experimental programmes. Chilton, NRPB-R266. London: HMSO, 1993.

5 Institute of Medicine. The Five Series Study: Mortality of military participants in US nuclear weapons tests. Washington, DC: National Academy Press, 2000

6 Pearce N, Winkelmann R, Kennedy J, et al. Further follow-up of New Zealand participants in United Kingdom atmospheric nuclear weapons tests in the Pacific. Cancer Causes Control 1997:8:139-45.

7 Rabbitt Roff S. A long time coming. New Scientist 6 February 1999

8 Rabbitt Roff S. Mortality and morbidity of members of the British Nuclear Tests Veterans Association and the New Zealand Nuclear Tests Veterans Association and their families. Medicine, Conflict and Survival 1999;15(suppl 1):1-51.

9 Muirhead CR, Bingham D, Haylock RGE, et al. Mortality and cancer incidence 1952-1998 in UK participants in the UK atmospheric nuclear weapons tests and experimental programmes. Chilton, NRPB-W27, 2003. Available from www.nrpb.org.

10 World Health Organisation. International Classification of Diseases, injuries and causes of death. 9th revision. Geneva: WHO, 1977.

11 Quinn MJ. Progress on flagging cancers at NHSCR. The Researcher 2000;1 14:4-5

12 Cartwright RA, Alexander FE, McKinney PA, et al. Leukaemia and lymphoma: an atlas of distribution within areas of England and Wales 1984-1988. London: Leukaemia Research Fund, 1990.

13 Breslow NE, Day NE. Statistical methods in cancer research. Volume II, The design and analysis of cohort studies. Scientific Publication No. 82. Lyon: International Agency for Research on Cancer, 1987.

14 United National Scientific Committee on the Effects of Atomic Radiation. Sources and effects of ionizing radiation. UNSCEAR 2000 Report to the General Assembly, with scientific annexes. New York: United Nations, 2000

15 Schottenfeld D, Fraumeni JF, eds. Cancer epidemiology and prevention 2nd edn. Oxford: Oxford University Press, 1996.

16 Preston DL, Kusumi S, Tomonaga $M$, et al. Cancer incidence in atomic bomb survivors. Part III: Leukemia, lymphoma and multiple myeloma 1950-1987. Radiat Res 1994;137:S68-97.

17 Cardis E, Gilbert ES, Carpenter L, et al. Effects of low doses and low dose rates of external ionizing radiation: cancer mortality among nuclear industry workers in three countries. Radiat Res 1995;142:117-32.

18 Muirhead CR, Goodill AA, Haylock RGE, et al. Occupational radiation exposure and mortality: second analysis of the National Registry for Radiation Workers. J Radiol Prot 1999;19:3-26.

19 Shimizu Y, Pierce DA, Preston DL, et al. Studies of the mortality of atomic bomb survivors. Report 12, Part II. Noncancer mortality: 1950-1990. Radiat Res 1999;152:374-89. 